\title{
ENVELHECER COM DEFICIÊNCIA FÍSICA: experiência com grupos educativos
}

\author{
Marineia Crosara de Resende* \\ Dóris Firmino Rabelo**
}

\section{Resumo}

O envelhecimento populacional brasileiro também está acontecendo entre as pessoas com deficiência física e as maneiras e condições pelas quais estas pessoas estão chegando à velhice, deve ser fonte de atenção por parte dos pesquisadores e das políticas públicas. Pretende-se apresentar uma experiência, realizada no segundo semestre de 2002 e no primeiro semestre de 2003 com os sócios da Associação dos Paraplégicos de Uberlândia, com grupos educativos envolvendo pessoas acima de 50 anos com deficiência física. Esta iniciativa objetivou contribuir para a preparação destas pessoas para um envelhecimento saudável, com mais qualidade de vida, trabalhando aspectos singulares do envelhecer com deficiência. O grupo "Qualidade de Vida e Velhice" aconteceu, semanalmente durante um ano, com a participação de três a quinze pessoas por encontro, e utilizou-se como recursos discussões, jogos e dinâmicas de grupo. A avaliação final dos grupos indicou que os participantes encontraram formas de melhorar sua qualidade de vida e atitudes frente à velhice.

Palavras-chave: Envelhecimento. Deficiência Física. Grupos. Gerontologia.

\section{Introdução}

Pensando na promoção da qualidade de vida e do bem-estar dos indivíduos mais velhos, especialmente aqueles com deficiência física, pretendemos com este texto apresentar como se deu a experiência com grupos educativos envolvendo pessoas que estão envelhecendo diante de incapacidades e limitações físicas congênitas ou adquiridas. Esperamos contribuir na busca de alternativas para o desenvolvimento do potencial de

* Psicóloga. Professora de Psicologia do Centro Universitário do Triângulo. Mestre em Gerontologia pela Universidade de Campinas (UNICAMP). Doutoranda em Educação: Gerontologia pela UNICAMP. E-mail: marineiaresende@terra.com.br

** Psicóloga. Mestranda em Gerontologia pela UNICAMP. E-mail: drisrabelo@yahoo.com.br 
idosos com algum tipo de deficiência física e na ampliação dos horizontes para a área do envelhecimento, que se apresenta como campo de atuação para diferentes profissionais, através do contato com ações políticas, sociais e pessoais voltadas à velhice, bem como estimular outros estudos e trabalhos de intervenção envolvendo esta temática.

A velhice e o processo de envelhecimento vêm sendo alvo de interesse de um número cada vez maior de profissionais, tendo em vista que o aumento no número e na proporção de idosos na população brasileira acarreta vários desafios nos âmbitos das políticas públicas, dos setores de saúde, de assistência social, educacional, da família e da formação de recursos humanos. Com isto cresce a preocupação e a investigação dos aspectos relativos à qualidade de vida na velhice e das condições que levam ao bom envelhecimento na tentativa de compreender as peculiaridades e necessidades desta população emergente e para a geração de alternativas de intervenção. (FREIRE, 2001; RODRIGUES; RAUTH, 2002).

Dentre o contingente da população brasileira que está envelhecendo tem-se um número crescente de pessoas com deficiência física, que estão nesse processo. A qualidade de vida dessas pessoas começa também a despertar a atenção de pesquisadores e profissionais que atuam diretamente com questões relacionadas às deficiências, em parte por causa do aumento da possibilidade dessas pessoas viverem por mais tempo, graças aos avanços da medicina, em parte por causa dos progressos sociais relativos à consideração dos direitos de pessoas com deficiência. (RESENDE, 2001).

Segundo Neri (2004) são muitos os campos em que a Psicologia pode contribuir para o bem-estar e a qualidade de vida dos idosos. Dentre estes destacam-se as atividades grupais como os centros de convivência e programas educativos, que visam o aprimoramento de habilidades individuais e sociais. Os psicólogos teriam muito a oferecer quando se trata dos indivíduos na chamada "situação de vulnerabilidade social", no qual está enquadrada a deficiência física. Nessa oferta de serviços a idosos, um psicólogo especializado poderia desenvolver planejamento e execução de intervenção psicológica e assessoria em programas de instituições públicas e privadas.

Atualmente, crescem as ações voltadas para os grupos de convivência de idosos, com ou sem deficiência física, e os grupos educativos que preparam as pessoas para envelhecer bem, contribuindo para a promoção da qualidade de vida destes indivíduos. São vários os argumentos favoráveis aos trabalhos educacionais, da promoção, da integração e da participação dos idosos à 
vida social. Além de proporcionarem novas aprendizagens, são importantes recursos para manter a funcionalidade, a adaptação dos idosos, as trocas sociais e a possibilidade de crescimento pessoal. Estas condições são necessárias para se chegar bem à velhice. (FREIRE, 2003).

Em ações educativas realizadas em grupo, particularmente nos grupos com pessoas com características peculiares, como é o caso das pessoas com deficiência física, é possível emergir mecanismos como: oferecimento de informações, instilação de esperança, universalidade, comportamento imitativo, altruísmo, desenvolvimento de técnicas de socialização, aprendizagem interpessoal e coesão. (VINOGRADOV; YALOM, 1992). Como funciona cada um desses mecanismos em relação à grupos educativos ou de convivência de pessoas que estão envelhecendo com deficiência física, com base nos autores acima, está explicitado a seguir.

$\mathrm{O}$ oferecimento de informações, função central em um grupo educativo, ocorre sempre que o coordenador oferece instrução didática acerca do funcionamento físico ou mental, e é possível oferecer orientação diretamente sobre as questões relacionadas ao envelhecimento, à deficiência física e aos fatores que são considerados importantes para se chegar à velhice da melhor maneira possível.

A instilação de esperança se apresenta quando a crença na efetividade do grupo fica evidente, ou seja, as pessoas buscam o grupo na intenção de usufruírem de algum benefício, mesmo que subjetivo. Uma pessoa pode se sentir estimulada por seu progresso, mas também pelo progresso do outro e considerar que se o outro se beneficia, ela também pode se beneficiar. A universalidade possibilita às pessoas descobrirem no grupo que não estão sós em sua condição de conviverem com limitações físicas, mas que outras pessoas também convivem com algumas desvantagens em função da deficiência.

Quando o comportamento imitativo, outro mecanismo atuante, está presente, os membros do grupo beneficiam-se da observação dos outros componentes com problemas similares, e passam a imitar aqueles comportamentos que dão certo. Nesse aspecto, o altruísmo também ocorre, e os participantes passam pela experiência de serem úteis aos outros membros do grupo, compartilhando problemas similares e oferecendo apoio, o que gera o desenvolvimento de técnicas de socialização. No grupo, criam-se oportunidades de vivenciarem técnicas de integração e socialização que podem ser expandidas, refletindo nos relacionamentos fora do grupo. 
A aprendizagem interpessoal, corresponde ao feedback que a pessoa recebe de seus colegas, informando-lhes dados a seu respeito. Desta forma tem chance e liberdade de fazer as correções necessárias para seu ajustamento. Os membros começam a perceber que existe um limite para a orientação e apoio que podem receber dos outros. Podem vir a descobrir que os maiores responsáveis por sua autonomia e pela condução de suas vidas são eles próprios.

E por último, a coesão do grupo, elemento fundamental para seu bom funcionamento, ocorre quando os membros de um grupo aceitam uns aos outros, oferecem apoio e estão inclinados a formarem relacionamentos significativos dentro do grupo. A coesão do grupo também oferece condições de aceitação e compreensão, favorece as revelações íntimas, o assumir riscos e a manutenção construtiva da confrontação e conflito.

Nesta perspectiva, a interação e as atividades de grupos com pessoas da própria geração e com experiências semelhantes, favorecem o bem-estar do indivíduo, trazendo como conseqüência a construção de significados comuns, a maior troca de experiências e contatos sociais e a ocorrência de catarse. Estas iniciativas propiciam a manutenção da flexibilidade, da funcionalidade, do aperfeiçoamento pessoal, o engajamento social e o bemestar subjetivo. (NERI; CACHIONI, 1999). Segundo Santos e Sá (2000), os programas educacionais para idosos são importantes para o desenvolvimento de atitudes de superação de dificuldades, de oportunidades de busca de seu bem-estar e de vitória sobre os desafios impostos pelas limitações físicas e pela sociedade no que diz respeito aos estereótipos e preconceitos. Estes programas, portanto, trazem a possibilidade de conquistar mais qualidade de vida e um bom envelhecimento.

As possibilidades de se envelhecer bem na presença de deficiência física, estão associadas à idéia de realização do potencial do indivíduo dentro de seus limites, na tentativa de alcançar bem-estar físico, social e psicológico, e também com o mecanismo de seleção e otimização de domínios que o idoso tem melhor nível de funcionamento, garantindo a compensação de perdas. Sendo assim, o importante é que este potencial e a utilização de mecanismos associados ao envelhecer satisfatoriamente sejam explorados adequadamente, e os grupos podem se tornar um meio interessante na busca desse fim. (NERI; CACHIONI, 1999).

Nesse sentido, envelhecer é uma experiência plural e não precisa necessariamente estar acompanhada de perdas, doenças e afastamento social. 
A possibilidade de uma velhice satisfatória é fato, mesmo na presença de incapacidades, pois as pessoas podem equilibrar suas limitações com suas potencialidades. (NERI, 2001). A maneira e as condições pelas quais estas pessoas estão chegando à velhice merecem atenção por parte dos pesquisadores, profissionais e das políticas públicas, visto que a população idosa vem aumentando. A velhice e a deficiência física ainda povoam a mente humana com pré-conceitos e tabus que vêm sendo perpetuados ao longo dos anos, tais como o mito da dependência e da improdutividade. (RESENDE, 2001).

Desta forma, tendo em vista a necessidade de pesquisas e intervenções no que tange à diversidade inerente à realidade da velhice e, dos benefícios já observados que os programas educacionais podem trazer a esta população, acredita-se ser de fundamental importância proporcionar espaços educacionais e de convivência para pessoas que estão envelhecendo com deficiência física. Trabalhos desta natureza, que visem quebrar tabus e preconceitos, incentivar as pessoas a desenvolverem suas capacidades, levando-as a verem a velhice sob outros ângulos, fazem parte dos esforços que devem ser empreendidos na promoção de mais qualidade de vida a estas pessoas.

Sendo assim, com base nas idéias colocadas anteriormente, foi desenvolvido um trabalho educativo em grupo, intitulado "Qualidade de Vida e Velhice", envolvendo pessoas acima de 50 anos associadas à uma instituição de pessoas com deficiência física da cidade de Uberlândia, Minas Gerais. Com esta iniciativa pretendeu-se contribuir na preparação de pessoas com deficiência física para um envelhecimento saudável, com mais qualidade de vida, trabalhando aspectos singulares do envelhecer com deficiência, ajudando-as a fortalecerem os mecanismos de autoregulação do self e trabalhando suas atitudes em relação a velhice e a deficiência física.

\section{Caracterização e Planejamento do Grupo}

A Associação dos Paraplégicos de Uberlândia ${ }^{1}$ (APARU), trabalha com questões relacionadas à deficiência física desde 1979 e tem entre seus principais objetivos promover as pessoas com deficiência física no tocante ao respeito e convivência, promoção humana, capacitação profissional,

\footnotetext{
1 Ressalta que o nome da instituição faz parte da história de Uberlândia e que portanto não acompanhou a evolução nas denominações relativas à deficiência física, sendo que em seu estatuto o termo paraplégico aparece apenas no nome da instituição e os associados são denominados pessoas com deficiência física.
} 
habilitação e reabilitação, atividades físicas e desportivas e à cidadania. Conta com aproximadamente mil e oitocentos associados freqüentes, para os quais oferece atendimento em vários setores, como psicologia, serviço social, odontologia, fisioterapia, jurídico, entre outros para que a inclusão seja realidade em Uberlândia. (RESENDE, 2004).

Nesta entidade, acontecem reuniões mensais com todos os associados a fim de possibilitar que as pessoas sejam mais importantes que as deficiências, sendo desenvolvidos palestras e trabalhos educativos. A proposta do grupo "Qualidade de Vida e Velhice" foi divulgada durante uma dessas reuniões mensais, resultando em dois grupos formados por pessoas acima de cinqüenta anos associadas à instituição. O grupo aconteceu semanalmente, com a participação de três a quinze pessoas por encontro, por dois semestres, sendo a idade média dos participantes 58 anos, a maioria mulher (90\%), casada (30\%) ou viúva (30\%), com escolaridade de $1^{\mathrm{a}}$ a $4^{\mathrm{a}}$ séries $(80 \%)$ e não aposentadas (90\%). Dentre estes, 30\% participam de outros grupos.

O planejamento do conteúdo a ser trabalhado no início de cada semestre foi participativo. Os encontros que deveriam integrar o projeto de trabalho contemplaram o interesse e o modo de vida dos participantes, valorizando suas experiências pessoais e diferenças interindividuais. Para levantamento das necessidades do grupo, primeiramente foi aberto um espaço na reunião semanal para discussão dos interesses, curiosidades e necessidades dos participantes.

Para levantamento das crenças sobre velhice e deficiência física foi feita uma técnica de colagem de figuras que representassem a velhice e a deficiência física. Pediu-se para que os participantes procurassem em revistas, todas as figuras que de alguma forma se associavam a estas duas realidades. Com estas figuras, deveriam fazer uma montagem em cartolina. Em seguida, cada pessoa mostrava e explicava sua produção. A partir disso, foram feitas discussões sobre como é envelhecer com deficiência física e como a realidade da velhice é diversificada nos contextos sócio-histórico-culturais. Foram também aplicados instrumentos com o objetivo de investigar os motivos que levaram os adultos a participarem do grupo e quais são suas concepções de velhice.

Verificou-se a partir desses dados que o que levou estas pessoas a procurar o grupo refere-se ao interesse de saber como envelhecer bem, pelo envolvimento social, pela vontade de aprender coisas novas e para saírem de casa e aproveitarem o que não puderam antes. As atitudes em relação à 
velhice foram negativas quando se trata da velhice pessoal, de uma vida sexual satisfatória nesta fase, da angústia associada a esta etapa, a questão do lazer e descanso e da possibilidade de satisfação na velhice. Velhice foi definida como uma palavra de conotação negativa por $80 \%$ dos sujeitos. Os pontos positivos da velhice considerados foram a experiência, a aposentadoria, o amor da família e a amizade. Os pontos negativos referem-se ao abandono, ao desemprego, à incapacidade, aos maus-tratos, à solidão, à morte, às doenças e ao preconceito. Os dados indicaram que as concepções de velhice foram, em sua maioria, negativas e que estas deveriam servir como subsídios dos aspectos a serem considerados durante o trabalho do grupo.

Os participantes demonstraram interesse em discutir os temas das limitações físicas, experiência de vida, alimentação, memória, autoconfiança e automotivação, como "não ser chato e cheio de manias na velhice". Relataram que gostariam que outros temas fossem preparados pelos facilitadores.

Dessa forma foram planejados os encontros, priorizando os interesses do grupo aliados à aprendizagem mediante dinâmicas de grupo, discussões, jogos e atividades lúdicas de caráter educativo em detrimento da escrita e leitura.

\section{Os Encontros e Avaliação do Grupo "Qualidade de Vida e Velhice"}

No segundo semestre de 2002 foram realizadas nove reuniões, com temas relativos ao desenvolvimento humano, aos mitos relacionados à velhice, às necessidades humanas básicas, ao sentido e metas de vida, criatividade, nutrição, memória, felicidade e ao envelhecimento bem sucedido. Durante esta fase os participantes relataram estarem ansiosos quanto à velhice pessoal e associaram esta fase à angústia, tristeza, desengajamento social e pouca possibilidade de satisfação e felicidade.

No primeiro semestre de 2003 foram realizadas dez reuniões nas quais se trabalhou temas como ansiedade; autoestima e autoimagem; mitos e fatos sobre a velhice; processos cognitivos na velhice; sentimentos, emoções e estratégias para lidar com elas embasadas na teoria sobre inteligência emocional. No início desta fase, novamente os participantes apresentaram uma considerável ansiedade quanto à autoaceitação da velhice.

Pode-se dizer que, de modo geral, houve uma dificuldade na aceitação da velhice pessoal, pois evidenciaram que não poderiam ainda ser 
considerados velhos uma vez que não estavam doentes e dependentes. Provavelmente este fato é um indicativo de que a palavra "velho" ainda carrega muitas conotações negativas e além disso nossa sociedade atual assiste a uma supervalorização daqueles adjetivos que estão mais próximos de pessoas jovens. Ao final de cada semestre, foi aberto um espaço para que os participantes fizessem uma avaliação do grupo. Estes disseram que participar das reuniões os ajudou muito na melhor compreensão da velhice apesar desta ainda parecer desafiadora dos seus limites, que se sentiam mais animados e dispostos na responsabilidade de buscar melhor qualidade de vida e foi muito importante para tornarem-se mais comunicativos com as pessoas. Os participantes manifestaram que ficou evidente que a deficiência física não é um fator ameaçador para eles (acredita-se que estão bem adaptados à sua condição de viver com uma deficiência física), diferentemente da velhice, da possibilidade de ficarem velhos.

Na avaliação final do trabalho realizado, procurou-se além dos relatos pessoais, investigar através de questionário, de que maneira participar do grupo influenciou no bem-estar e na mudança de percepção dos outros a respeito dos participantes. Consideraram que os aspectos que mais contribuíram para o seu bem-estar referem-se à melhora no relacionamento familiar e na sua capacidade de auto-afirmação (83,3\%). Acreditam que depois que começaram a freqüentar o grupo, o que mais mudou na percepção dos outros a seu respeito é a confiança na sua capacidade $(66,7 \%)$ e que a maior contribuição do grupo foi o apoio e entender que na velhice existe a possibilidade de desenvolvimento.

De modo geral, os participantes dos dois grupos salientaram ao longo dos encontros que a experiência de participação nos grupos da instituição é fonte de bem-estar e motivação. Um dado comum observado nos dois grupos foi a necessidade, por parte de alguns membros, de dar uma moldura terapêutica para os conflitos pessoais e familiares que eram trazidos para os encontros. Os coordenadores, sem perder a orientação do objetivo do grupo, pontuaram algumas das necessidades trazidas mediante enfoque terapêutico diretivo.

\section{Considerações Finais}

Acredita-se que os objetivos deste trabalho foram alcançados. A partir da avaliação final dos grupos, que relataram ter encontrado formas de 
melhorar sua qualidade de vida e atitudes frente à velhice, e pelas observações do desenvolvimento dos encontros, verificou-se que estas pessoas começaram a pensar a velhice por um outro ângulo, agora não somente o das perdas, mas o da possibilidade de desenvolvimento e crescimento humano contínuo, ao longo de todo o curso de vida.

Esse tipo de trabalho é importante uma vez que estimula os participantes no comprometimento de buscarem formas para um bom envelhecimento dentro dos limites de cada um. Sendo assim, trabalhos educativos propostos dentro desta temática são necessários na medida em que possibilitam que pessoas envelhecendo em contextos diferentes, desenvolvam meios para um maior senso de bem-estar subjetivo e uma melhor qualidade de vida.

Para algumas pessoas com deficiência física, as perdas inerentes ao processo de envelhecimento acumulam-se sobre as desvantagens decorrentes de eventos ocorridos em fases precedentes. No entanto, Resende (2001) afirma que as limitações impostas pela deficiência são superadas e as pessoas se adaptam às suas condições, pautadas nas circunstâncias de vida e na influência de fatores como a personalidade, as metas de vida, o temperamento do indivíduo, bem como de fatores ambientais e da competência comportamental.

Envelhecer bem com deficiência física requer um ajustamento pessoal e social, principalmente sob condições deficitárias de saúde e educação, realidade em que vive a maioria da população de todas as idades dos países em desenvolvimento. A participação em grupos educativos possibilita o ajustamento psicossocial, uma vez que as estratégias de enfrentamento dependem de recursos pessoais, e também de recursos sociais, para envelhecer com boa qualidade de vida.

\title{
AGING WITH DISABILITY: experience with educational groups
}

\begin{abstract}
The populational ageing of Brazilian with physical disability and the conditions with those are coming to elder should be source of attention of researchers and public policies. This text intends to present an experience
\end{abstract}


realized with Uberlândia's Paraplegics Association's members, at the period of the second semester of 2002 and the first semester of 2003, with educational groups involving people above 50 years with physical disability. This initiative intended to contribute for these people's preparation for a healthy aging, with more life quality, working singular aspects of aging with deficiency. The group "The Age Process and Quality of Life" weekly happened for one year, with the participation from three to fifteen people for meeting, and the used resources were discussions, games and group dynamics. It is believed that the objectives of this work were reached, starting from the final evaluation of the groups, that the participants told to have found forms of improving their life quality and attitudes toward aging.

Keywords: Aging. Physical Disability. Groups. Gerontology.

\section{REFERÊNCIAS}

FREIRE, S. A. Bem-Estar Subjetivo e Metas de Vida: um estudo transversal com homens e mulheres pertencentes a três faixas de idade. 2001. Tese (Doutorado em Educação) - Universidade Estadual de Campinas, Campinas, 2001 .

FREIRE, S. A. Educação Permanente e Qualidade de Vida na Velhice: metas e desafios na atualidade. In: CONGRESSO PAULISTA DE GERIATRIA E GERONTOLOGIA, 3., 2003, Santos. Anais... Santos: SBGG-SP, 2003. 1 CD-ROM.

NERI, A. L. Velhice e Qualidade de Vida na Mulher. In: NERI, A. L. Desenvolvimento e Envelhecimento: perspectivas biológicas, psicológicas e sociológicas. Campinas: Papirus, 2001. P. 161-200.

NERI, A. L. O que a Psicologia tem a Oferecer ao Estudo e à Intervenção no Campo do Envelhecimento no Brasil, Hoje. In: NERI, A. L.; YASSUDA, M. S. (Org.); CACHIONI, M. (Colab.). Velhice Bem-Sucedida: aspectos afetivos e cognitivos. Campinas: Papirus, 2004. P. 13-28. 
NERI, A. L.; CACHIONI, M. Velhice Bem-Sucedida e Educação. In: NERI, A. L.; DEBERT, G. G. (Org.). Velhice e Sociedade. Campinas: Papirus, 1999. P. 113-140.

RESENDE, M. C. Atitudes em Relação ao Idoso, à Velhice Pessoal e ao Portador de Deficiência Física em Adultos Portadores de Deficiência Física. 2001. 89 f. Dissertação (Mestrado em Gerontologia) - Universidade Estadual de Campinas, Campinas, 2001.

RESENDE, A. P. C. Todos na Cidade: o direito a acessibilidade das pessoas com deficiência física em Uberlândia. Uberlândia: EDUFU, 2004.

RODRIGUES, N. C.; RAUTH, J. Os Desafios do Envelhecimento no Brasil. In: FREITAS, E. V.; NERI, A. L.; PY, L.; CANÇADO, F. A. X.; GORZONI, M. L.; ROCHA, S. M. (Org.). Tratado de Geriatria e Gerontologia. Rio de Janeiro: Guanabara Koogan, 2002. P. 106-111.

SANTOS, A. T.; SÁ, M. A. A. S. De Volta às Aulas: ensino e aprendizagem na terceira idade. In: NERI, A. L.; FREIRE, S. A. E por Falar em Boa Velhice. Campinas: Papirus, 2000. P. 91-100.

VINOGRADOV, S.; YALOM, I. D. Manual de Psicoterapia de Grupo. Porto Alegre: Artes Médicas, 1992. 